\title{
Relapsing fever in young refugees from East Africa
}

\author{
Spinello Antinori ${ }^{1,2^{*}}$ (D) Valeria Colombo ${ }^{1}$ and Mario Corbellino ${ }^{1,2}$ \\ See related letter by Cutuli et al. https://ccforum.biomedcentral.com/articles/10.1186/s13054-017-1666-5
}

We read with interest the letter by Cutuli et al. [1] describing a case of severe co-infection by Leptospira spp. and Borrelia recurrentis in a young female refugee from East Africa. We would like to comment on several issues raised by their paper.

First, the authors state "nits were present on her scalp...". In our opinion, this sentence may mislead readers as meaning that "head lice" (Pediculus humanus capitis) are the vectors of louse-borne relapsing fever (LBRF). Indeed, body lice (Pediculus humanus humanus) are to date the only demonstrated vectors of the disease. We are aware of only two reports providing proof that head lice can harbor $B$. recurrentis and consequently might act as a vector of LBRF [2,3]. However, in the study conducted in Ethiopia, body lice had concomitantly infested the patients and thus the authors could not formally exclude that the latter were not implicated as vectors of the disease [2].

The second point that needs clarification is the relative contribution of the two microorganisms (i.e., Leptospira and Borrelia) to the described clinical picture: shock, acute respiratory distress syndrome (ARDS), renal failure, jaundice, anemia, thrombocytopenia, altered blood coagulation, and high procalcitonin. With the exclusion of ARDS, which may correctly be attributed to severe leptospirosis, all other findings can be observed in patients with severe clinical presentation of both diseases. However, in the recent wave of LBRF observed in Europe, intensivists were faced with severe cases of LBRF presenting with shock solely as a consequence of the Jarisch-Herxheimer reaction precipitated by administration of antibiotics [4].

A final point that deserves comment concerns the microbiology diagnosis. Molecular biology by means of real-time polymerase chain reaction in this case provided the correct diagnosis of both infections. Although not clearly stated, the authors report that malaria was excluded among other possible differential diagnoses. Thus, we can infer that blood smears examined were negative not only for Plasmodium species but also for Borrelia. This fact may hint at the possibility that the patient suffered from a relapse and not a first episode of LBRF although Cutuli et al. state that "she had no relevant past medical history". Given the linguistic barrier encountered with refugees, a previous episode might have been overlooked. Finally, although LBRF was not mentioned in a review dealing with diagnosis and management of critically ill migrants [5], intensivists should be aware of the increasing frequency of this infectious disease.

\section{Authors' response}

\section{Salvatore L. Cutuli, Gennaro De Pascale, Alessandra Ciervo and Massimo Antonelli}

We are pleased to answer the issues raised by Antinori and coworkers over the case of a young critically ill female refugee who developed ARDS and multi-organ dysfunction (MOD) associated with $B$. recurrentis and Leptospira species co-infection [6].

\footnotetext{
*Correspondence: spinello.antinori@unimi.it

'Department of Biomedical and Clinical Sciences "Luigi Sacco", Università degli Studi di Milano, Via GB Grassi, 74, 20157 Milano, Italy

${ }^{2}$ III Division of Infectious Diseases, ASST Fatebenefratelli Sacco, Milano, Italy
}

First, we confirm that the patient had plenty of body lice but the nits were first identified on her scalp. Second, we cannot identify the specific role of the single agent (Borrelia or Leptospira) in the severe clinical picture, although this was not meaningful from a clinical point of view. Lastly, we cannot exclude that the patient suffered from a relapse rather than a first episode of Borrelia infection, and the information we retrieved 
from her relatives (who could speak French), were not useful for clarifying this issue.

We are grateful to Antinori and coworkers for their interesting feedback. We think that our experience witnesses the tricky role of medical staff dealing with a life-threatening clinical picture, re-emerging diseases, and difficult health care organization.

Collaboration among infectious disease specialists, microbiologists, and ICU physicians may be key to solving this issue.

\section{Abbreviations}

ARDS: Acute respiratory distress syndrome; LBRF: louse-borne relapsing fever

\section{Acknowledgements}

None.

\section{Funding}

The study did not receive any funding.

\section{Availability of data and materials}

The datasets used and/or analyzed during the current study are available from the corresponding author on reasonable request.

\section{Authors' contributions}

SA and MC conceived the study and wrote the manuscript; SA and VC analyzed the literature. SA, VC, and MC reviewed and approved the final version of the manuscript. As the corresponding author, SA has full access to all the data in the study and has final responsibility for the decision to submit for publication. All authors read and approved the final manuscript.

Ethics approval and consent to participate

Not applicable. All data were reported retrospectively.

\section{Consent for publication}

Not applicable. All data were collected and reported anonymously. The patient ran away from the hospital and never came back to our ICU followup clinic.

\section{Competing interests}

The authors declare that they have no competing interests.

\section{Publisher's Note}

Springer Nature remains neutral with regard to jurisdictional claims in published maps and institutional affiliations.

Published online: 03 August 2017

\section{References}

1. Cutuli SL, De Pascale G, Spanu T, Dell'Anna AM, Bocci MG, Pallavicini F, et al. Lice, rodents, and many hopes: a rare disease in a young refugee. Crit Care. 2017;21:81.

2. Boutellis A, Mediannikov O, Bilcha KD, Ali J, Campelo D, Barker SC, et al. Borrelia recurrentis in head lice. Ethiopia Emerg Infect Dis. 2013;19:796-8.

3. Amanzougaghene N, Akiana J, Ndombe GM, Davoust B, Nsana NS, Parra H$J$, et al. Head lice of pygmies reveal the presence of relapsing fever Borreliae in the Republic of Congo. Plos Neglect Trop Dis. 2016;10(12):e0005142.

4. Antinori S, Mediannikov O, Corbellino M, Raoult D. Louse-borne relapsing fever among Easr African refugees in Europe. Travel Med Infect Dis. 2016;14:110-4.

5. Poulaku G, Bassetti M, Timsit J-F. Critically ill migrants with infection: diagnostic considerations for intensive care physicians in Europe. Intensive Care Med. 2016;42:245-8.

6. Cutuli SL, De Pascale G, Spanu T, Dell'Anna AM, Bocci MG, Pallavicini F, et al. Lice, rodents, and many hopes: a rare disease in a young refugee. Critical Care. 2017:21:81. 